Published in final edited form as:

Int Urol Nephrol. 2016 January ; 48(1): 73-77. doi:10.1007/s11255-015-1145-3.

\title{
Metastatic renal cell carcinoma without evidence of a renal primary
}

\author{
Corey Costantino ${ }^{1,2}$, George V. Thomas ${ }^{2,3}$, Christopher Ryan ${ }^{3}$, Fergus V. Coakley ${ }^{4}$, and \\ Megan L. Troxell 2,3 \\ Megan L. Troxell: troxellm@ohsu.edu \\ ${ }^{1}$ School of Medicine, Oregon Health \& Science University, Portland, OR, USA \\ ${ }^{2}$ Department of Pathology, Oregon Health \& Science University, 3181 SW Sam Jackson Park Rd, \\ L418, Portland, OR 97239, USA \\ ${ }^{3}$ Knight Cancer Institute, Oregon Health \& Science University, Portland, OR, USA \\ ${ }^{4}$ Department of Diagnostic Radiology, Oregon Health \& Science University, Portland, OR, USA
}

\begin{abstract}
Purpose-Metastatic renal cell carcinoma (RCC), without an identified kidney primary, has been reported rarely. We report a patient with RCC metastatic to bilateral adrenal glands and liver, without an apparent renal primary. We detail the immunohistochemical and molecular studies employed to substantiate the diagnosis of RCC and direct therapy.
\end{abstract}

Methods-Histopathologic findings were correlated with imaging data and supplemented by a panel of immunohistochemical stains, as well as tumor sequence analysis.

Results-Despite the presence of bilateral adrenal masses and lack of tumor within kidney parenchyma, the diagnosis of RCC was substantiated by immunohistochemistry (RCC+/PAX2+/ PAX8+/Melan-A-/SF-1- among others) and molecular genetic analysis, harboring mutations in $V H L, T P 53, K D M 5 C$, and PBRM1. After debulking surgery, based on the diagnosis of RCC and the molecular profile, the patient was treated with a tyrosine kinase inhibitor (sunitinib), resulting in stablilization of disease.

Conclusions-This case illustrates the role of mutational analysis in carcinomas with rare or unusual presentations, such as metastatic RCC without a renal primary.

\section{Keywords}

Renal cell carcinoma; Adrenal cortical carcinoma; Sunitinib; VHL

\section{Introduction}

Renal cell carcinoma (RCC) can metastasize to almost any organ, with the most common sites being lung, liver, bones, and brain, and $\sim 25 \%$ of RCC patients will have distant

\footnotetext{
Compliance with ethical standards

Conflict of interest The other authors declare that they have no conflicts of interest.
} 
metastases at the time of presentation [1,2]. Although a less common site of metastasis, RCC may present with synchronous ipsilateral, contralateral, and rarely bilateral adrenal metastasis [2]. There have been limited case reports of metastatic RCC presenting without evidence of an identifiable primary renal tumor [3-9], including one case report of metastatic RCC to the adrenal gland without an identifiable renal primary [9]. Herein, we report a case of RCC presenting with bilateral adrenal and liver metastases, as well as extensive tumor thrombus extending from the bilateral renal veins to the inferior vena cava, without evidence of a primary renal tumor.

\section{Case report}

A 68-year-old male presented with 12 months of fatigue, anorexia, postprandial nausea, and unintended 30-40 lb weight loss. Computed tomography scan of the abdomen and pelvis revealed bilateral adrenal masses (19.5-cm tumor in left adrenal and 10.7-cm tumor in right), each associated with tumor thrombus extending from the renal vein into the inferior vena cava (Fig. 1). Subsequent MRI demonstrated interval enlargement of both tumors, as well as an indeterminate segment 8 subcapsular hepatic lesion. A left adrenal mass core biopsy was performed and showed sheets and ribbons of cells with abundant eosinophilic cytoplasm and occasional prominent nucleoli; the immunophenotype was consistent with renal cell carcinoma (Table 1). The patient then underwent bilateral adrenalectomy, left nephrectomy, wedge resection of liver, IVC tumor thrombectomy with reconstruction, and retroperitoneal lymph node dissection. The left kidney was resected given adherence to the adrenal tumor, and involvement of the left renal vein. Pathology revealed metastastic poorly differentiated carcinoma, with similar morphology in bilateral adrenal and liver sections. The tumor was composed of malignant cells with eosinophilic cytoplasm and a high degree of nuclear pleomorphism, prominent nuclei, mitotic activity, and necrosis. The architecture was variable, including sheet-like areas, large glands, and papillary architecture (Fig. 2). In some areas, prominent cytoplasmic hyaline globules were present. Tumor cytology suggested either poorly differentiated adrenal cortical or high-grade renal cell carcinoma. The left nephrectomy specimen contained numerous simple cysts without excrescences, but no evidence of a renal tumor was identified despite thorough gross and histologic examination. No concerning renal masses in the right kidney were identified based on pre- or postoperative imaging. Immunohistochemical staining of the resection specimens again revealed an immunophenotype consistent with renal cell carcinoma (Table 1). Tumor mutation screening was performed and identified the following mutations in the tumor tissue: VHL (W88 fs*44), TP53 (R337H), KDM5C (R68*), PBRM1 (R1160*), KIT (P467S), and NOTCH1 (P1236L). Additional immunostaining for the VHL gene product was performed in multiple sections, all of which essentially demonstrated lack of VHL gene product expression, in keeping with the nonsense frameshift mutation uncovered by sequencing. Overall, the morphology, immunophenotype, and genetic profile of the tumor favored a diagnosis of high-grade renal cell carcinoma over adrenocortical carcinoma.

Subsequent imaging 4 months following surgical resection revealed rapid development of metastatic disease with a new peripancreatic aortocaval mass encasing the superior mesenteric artery and abutting the celiac trunk $(8.0 \times 3.8 \mathrm{~cm})$, an enlarged porta hepatis lymph node, a small (10 mm) liver lesion, and an enhancing T12 vertebral body lesion 
extending into the lamina. Based on the diagnosis of RCC and the molecular genetic finding of a VHL mutation, the patient was subsequently started on the tyrosine kinase inhibitor sunitinib $50 \mathrm{mg}$ per day (4 weeks on, 2 weeks off). Therapy was initially complicated by a hospital admission for fever and confusion of uncertain etiology, but sunitinib was subsequently resumed and was well tolerated. He received a course of palliative radiation therapy to the T12 lesion ( $30 \mathrm{~Gy}$ in 10 fractions). Six months after starting sunitinib, CT imaging revealed slight decrease in size of the aortocaval mass. The disease remains clinically stable 1 year after starting sunitinib.

\section{Discussion}

Renal cell carcinoma (RCC) has the potential to metastasize to almost any organ system and presents with synchronous metastases in about $25 \%$ of cases $[1,2,5]$. While the development of metastatic disease without an identifiable primary malignancy is extremely rare, there have been three well-documented cases of RCC presenting as metastatic disease without a renal primary, to our knowledge [5, 8, 9]. Johnson et al. [9] reported a case of metastatic RCC to the adrenal gland, with an associated involuted region of the ipsilateral kidney which was proposed to represent spontaneous regression of the primary tumor. Wayne et al. [5] reported a case of three metastatic foci of RCC (subcutaneous mass in the arm, with subsequent pancreatic and parotid gland metastases) without the identification of a renal primary. The diagnosis in both of these cases was supported by well-differentiated clear cell morphology and immunohistochemical staining. Heary et al. [8] reported a case of metastatic RCC arising in an epidural meningioma resection site 3 years after initial surgery, with no radiographic evidence of an occult renal primary. Herein, we report a case of poorly differentiated renal cell carcinoma that presented as bilateral adrenal and liver metastases without evidence of a renal primary. The diagnosis in the present case is supported by immunohistochemistry, but also by molecular genetic studies, highlighting the utility of such adjunct studies in differentiating high-grade tumors with overlapping morphology and immunophenotype.

Differentiating metastatic high-grade RCC from primary adrenal cortical carcinoma, especially in the rare instances of an absent primary tumor, can be difficult given the significant morphologic overlap between the two entities [10,11]. In cases of morphologic ambiguity, the utilization of immunohistochemistry and other adjunct studies becomes essential to arriving at the correct diagnosis. With the exception of focal inhibin staining, the overall immunohistochemistry profile in the present case is strongly in favor of RCC over adrenocortical carcinoma (Table 1). In their study investigating the immunohistochemical distinction between primary adrenal cortical lesions and metastatic clear cell renal cell carcinoma (ccRCC), Sangoi et al. [11] reported positive inhibin staining in $9 \%$ of metastatic ccRCC included in the study, including positive staining in $12 \%$ of the poorly differentiated ccRCC subgroup. Li et al. [10] compared 34 cases of metastatic ccRCC to 49 primary adrenal lesions and found carbonic anhydrase IX, RCC marker, and PAX8 to be the most sensitive and specific markers for ccRCC in this context. Papillary RCC shares this immunophenotype, but is often also positive for CK7, racemase, and napsin [12]. Despite focal inhibin staining in the present case, the overall immunohistochemical profile, including 
positive staining with RCC and PAX8, in addition to other traditional renal markers, strongly favors the diagnosis of RCC over adrenal cortical carcinoma.

The molecular genetics of RCC has been the subject of extensive investigation. Mutations in the von Hippel-Lindau gene $(V H L)$, a tumor suppressor gene whose discovery arose out of investigations into the pathogenesis of the inherited von Hippel-Lindau cancer syndrome, have also been frequently identified in non-hereditary ccRCCs and are believed to play a role in oncogenesis $[13,14]$. According to The Cancer Research Genome Atlas Network (TCGA) study involving molecular characterization of more than 400 nephrectomy specimens with known ccRCC, somatic mutations in the VHL gene were identified in 43.5 $\%$ of cases [15]. The same study identified PBRM1 and KDM5C mutations in $27.7 \%$ and $5.6 \%$ of cases, respectively [15]. In contrast, according to provisional data available through the TCGA, the respective mutation rates of $V H L, P B R M 1$, and $K D M 5 C$ were $0.0,1.1$, and $0.0 \%$, respectively, in a set of 88 patient cases of adrenocortical carcinoma $[16,17]$.

Although a comparison of the two data sets reveals that TP53 mutations tend to occur more frequently in adrenocortical carcinoma compared to ccRCC $(18.5 \%$ vs $1.8 \%)[16,17]$, functional loss of this tumor suppressor gene is common in many forms of aggressive advanced cancers [18]. The presence of multiple genetic mutations that are more characteristically seen in ccRCC compared to adrenocortical carcinoma, also argues strongly in favor of the diagnosis of RCC. This case also demonstrates the utility of molecular genetics in diagnosing ambiguous or poorly differentiated tumors.

In conclusion, metastatic RCC without an identifiable primary tumor is extremely rare. In this case, making the correct diagnosis of poorly differentiated RCC over high-grade adrenocortical carcinoma was particularly difficult given the distribution of disease at the time of presentation, and the overlapping histomorphology. This case once again demonstrates the diagnostic utility of immunohistochemistry in differentiating ambiguous cases. Additionally, this case provides a novel example where molecular genetic data were leveraged to support the correct diagnosis and guide molecularly targeted therapy that resulted in clinical benefit.

\section{Acknowledgments}

Funding Dr. Thomas receives support from NIH Grants R01 CA169172.

Dr. Ryan has received consulting fees from Pfizer.

\section{References}

1. Lau WK, Zincke H, Lohse CM, Cheville JC, Weaver AL, Blute ML. Contralateral adrenal metastasis of renal cell carcinoma: treatment, outcome and a review. BJU Int. 2003; 91:775-779. [PubMed: 12780830]

2. Özturk H. Bilateral synchronous adrenal metastases of renal cell carcinoma: a case report and review of the literature. Oncol Lett. 2015; 9:1897-1901. [PubMed: 25789064]

3. Marcus PB, Kemp CB. Ectopic renal cell carcinoma: pathologist's problem. Urology. 1978; 1978(12):453-457. [PubMed: 715974]

4. MacLennan, GT.; Cheng, L. Neoplasms of the Kidney. In: Bostwick, DG.; Cheng, L., editors. Urologic surgical pathology. 2nd. Mosby Elesvier: Maryland Heights; 2008. p. 83 
5. Wayne M, Wang W, Bratcher J, Cumani B, Kasmin F, Cooper-man A. Renal cell cancer without a renal primary. World J Surg Oncol. 2010; 8:18. [PubMed: 20302679]

6. Terada T. Extra-renal clear cell renal cell carcinoma probably arising from mesodermal embryonic remnants. Pathol Int. 2012; 62:291-293. [PubMed: 22449235]

7. Abdou AG, Kandil M, Elshakhs S, El-Dien MS, Abdallah R. Renal cell carcinoma with rhabdoid and sarcomatoid features presented as a metastatic thigh mass with an unusual immunohistochemical profile. Rare Tumors. 2014; 6:5037. [PubMed: 24711903]

8. Heary RF, Agarwal N, Barrese JC, Barry MT, Baisre A. Metastatic renal cell carcinoma, with a radiographically occult primary tumor, presenting in the operative site of a thoracic men-ingioma: long-term follow-up. J Neurosurg Spine. 2014; 21:628-633. [PubMed: 25014504]

9. Johnson MT, Bahnson RR, Zynger DL. Metastatic clear cell renal cell carcinoma to the adrenal gland with-out an identifiable primary tumor. Int J Urol. 2012; 19:92-93. [PubMed: 22146098]

10. Li H, Hes O, MacLennan GT, Eastwood DC, Iczkowski KA. Immunohistochemical distinction of metastases of renal cell carcinoma to the adrenal from primary adrenal nodules, including oncocytic tumor. Vichows Arch. 2015; 466:581-588.

11. Sangoi AR, Fujiwara M, West RB, Montgomery KD, Bonven-tre JV, Higgins JP, Rouse RV, Gokden N, McKenney JK. Immunohistochemical distinction of primary adrenal corti lesions from metastatic clear cell renal cell carcinoma: a study of 248 cases. Am J Surg Pathol. 2011; 35:678686. [PubMed: 21490444]

12. Truong LD, Shen SS. Immunohistochemical diagnosis of renal neoplasms. Arch Pathol Lab Med. 2011; 135:92-109. [PubMed: 21204715]

13. Gossage L, Eisen T, Maher E. VHL, the story of a tumour suppressor gene. Nat Rev Cancer. 2015; 15:55-64. [PubMed: 25533676]

14. Li L, Kaelin WG. New insights into the biology of renal cell carcinoma. Hematol Oncol Clin North Am. 2011; 25:667-686. [PubMed: 21763962]

15. The Cancer Genome Atlas Research Network. Comprehensive molecular characterization of clear cell renal cell carcinoma. Nature. 2013; 499(7456):43-49. [PubMed: 23792563]

16. Cerami E, Gao J, Dogrusoz U, Gross BE, Sumer SO, Aksoy BA, Jacobsen A, Byrne CJ, Heuer ML, Larsson E, Antipin Y, Reva B, Goldberg AP, Sander C, Schultz N. The cBio cancer genomics portal: an open platform for exploring multi-dimensional cancer genomics data. Cancer Discov. 2012; 2:401-404. [PubMed: 22588877]

17. Gao J, Aksoy BA, Dogrusoz U, Dresdner G, Gross B, Sumer SO, Sun Y, Jacobsen A, Sinha R, Larsson E, Cerami E, Sander C, Schultz N. Integrative analysis of complex cancer genomics and clinical profiles using the cBioPortal. Sci Signal. 2013; 6(269):11.

18. Brown CJ, Lain S, Verma CS. Awakening guardian angels: drugging the p53 pathway. Nat Rev Cancer. 2009; 9:862-873. [PubMed: 19935675] 


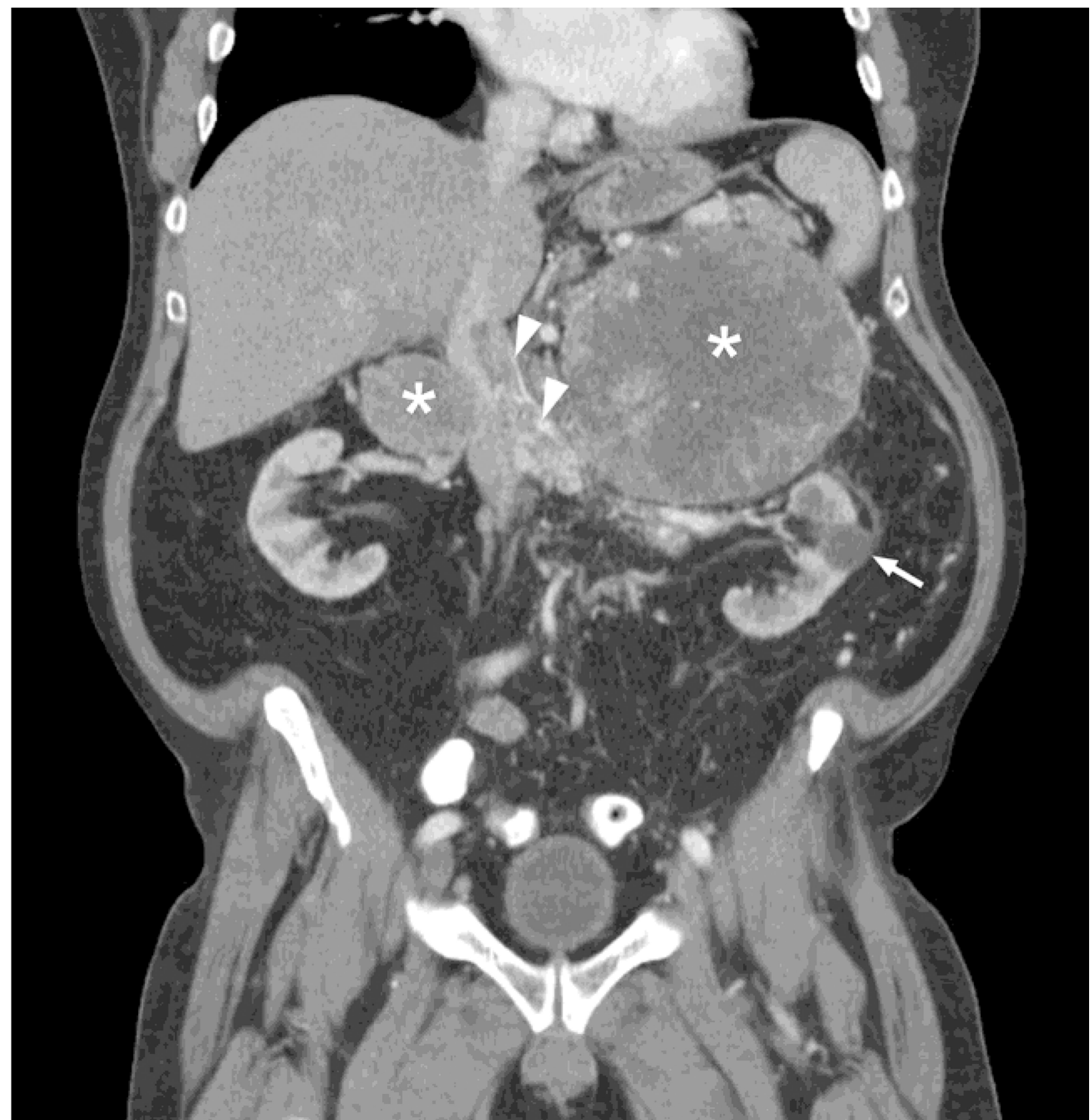

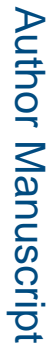

Fig. 1.

CT scan showing bilateral adrenal masses (asterisks) with associated tumor thrombus in the renal veins and inferior vena cava (arrowheads). Arrow denotes benign appearing simple cyst in left kidney 

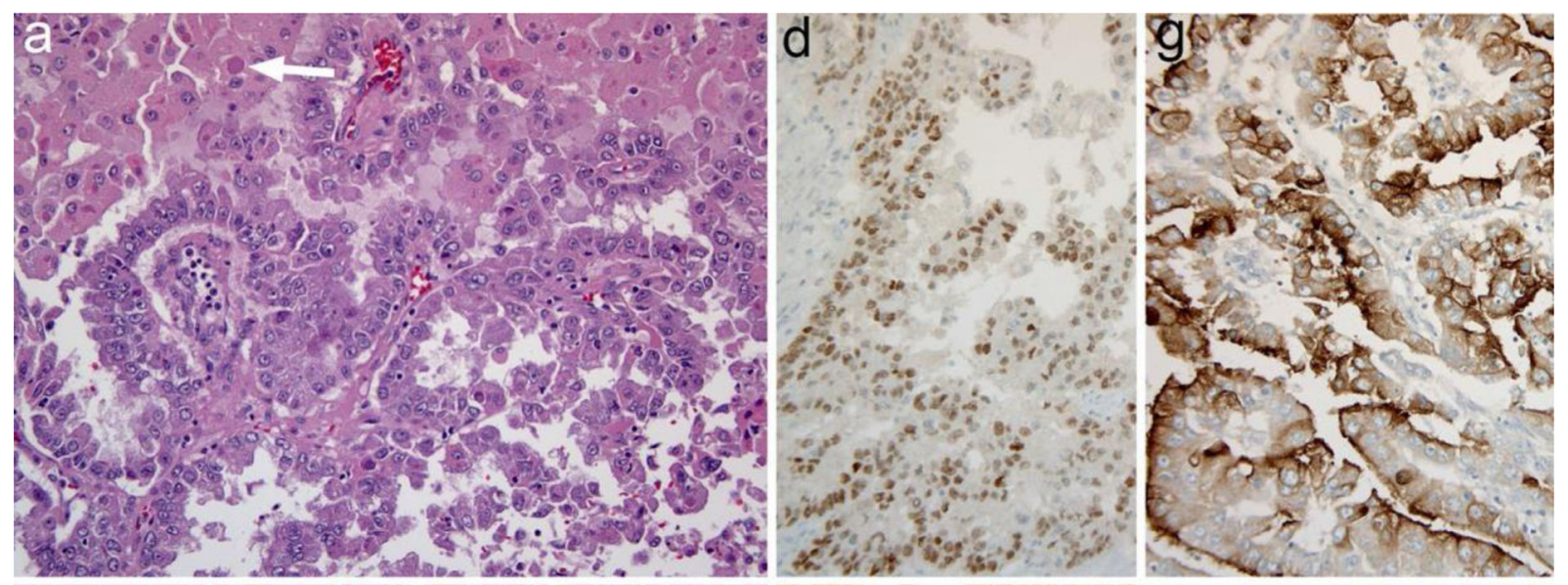

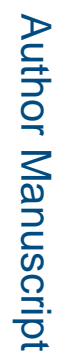
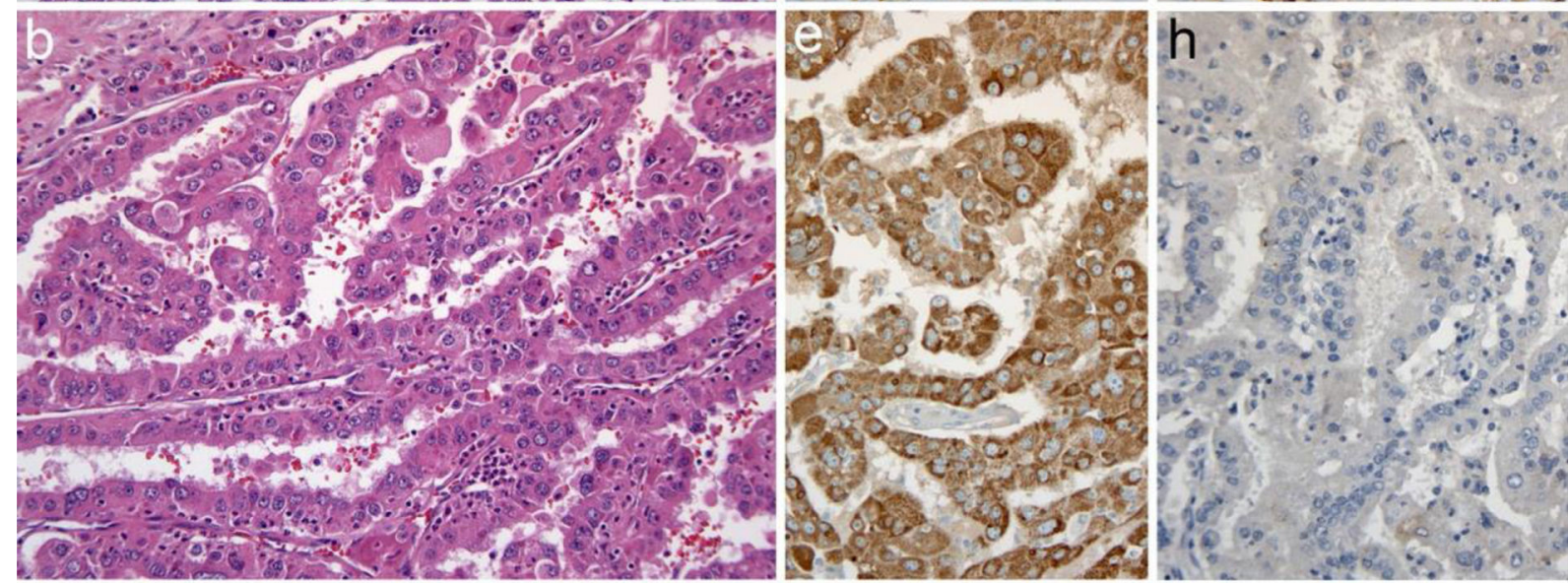

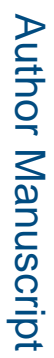
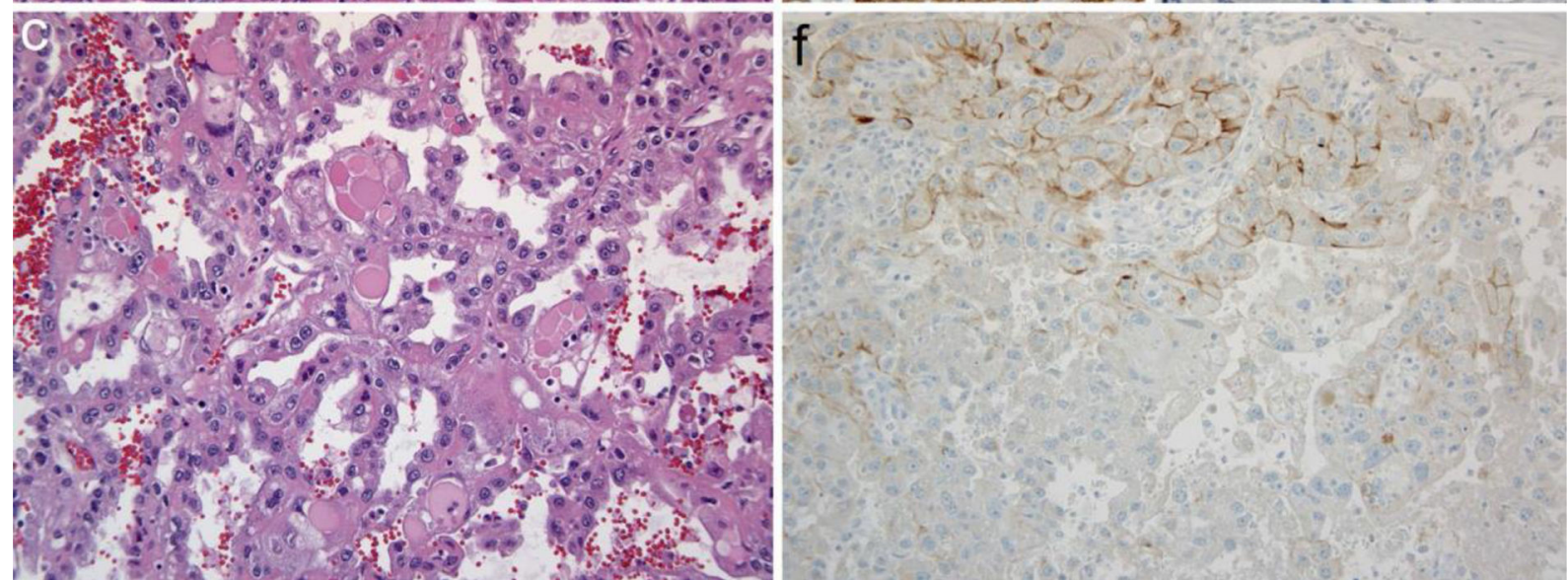

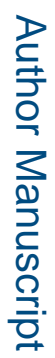

Fig. 2.

Tumor histology and immunohistochemistry a. Carcinoma from the left adrenal is composed of cells with eosinophilic cytoplasm, large nuclei, and prominent nuclei. Papillary architecture is shown at bottom, while cells at the top of the microscopic field have more abundant cytoplasm and some intracytoplasmic hyaline inclusions (arrow) b. Carcinoma from the liver metastasis has prominent papillary architecture and similar cytologic features; admixed neutrophils are present $\mathbf{c}$. Carcinoma from the right adrenal is again similar, but there are scattered very large cytoplasmic hyaline inclusions in this tumor focus d. PAX8 
immunohistochemical staining positive in tumor cells e. Racemase (AMACR, P504S) strongly diffusely positive in tumor cells $\mathbf{f}$. Immunostaining for the von Hippel-Lindau protein essentially negative; there was only weak cell membrane, or apical staining focally within the carcinoma, as shown in the top of the panel. The expected normal staining pattern diffuses cytoplasmic and membrane staining (not shown). g. RCC monoclonal antibody staining positive with the expected apical membrane accentuation. h. SF-1 immunohistochemistry negative. Original magnifications: 200x 


\section{Table 1}

Immunohistochemical profile

\begin{tabular}{|c|c|c|c|c|}
\hline Antibody stain & Adrenal biopsy & Left adrenal resection & Right adrenal resection & Liver \\
\hline \multicolumn{5}{|l|}{ Typical renal markers } \\
\hline RCC(renalcellcarcinomaantigen) & + & + & & \\
\hline PAX2 (paired box protein 2) & + & + & + & + \\
\hline PAX8 (paired box protein 8 ) & & + & + & + \\
\hline $\begin{array}{l}\text { CD10 (membrane pattern, also known as common acute } \\
\text { lymphoblastic leukemia antigen (CALLA) }\end{array}$ & & + & & \\
\hline Vimentin & + & & & \\
\hline $\begin{array}{l}\text { Racemase (also known as AMACR = alpha-methylacyl- } \\
\text { CoA racemase, } \mathrm{P} 504 \mathrm{~S} \text { ) }\end{array}$ & & + & & \\
\hline \multicolumn{5}{|l|}{ Typical adrenal markers } \\
\hline Inhibin & - & $+($ Focal $)$ & - & \\
\hline Melan-A & - & - & & \\
\hline SF-1(steroidogenic factor1 & & - & - & - \\
\hline Synaptophysin & - & - & & \\
\hline Calretinin & - & - & & \\
\hline \multicolumn{5}{|l|}{ Other } \\
\hline Pankeratin & + & + & + & \\
\hline CK CAM5.2 (CK = cytokeratin) & - & & & \\
\hline EMA (epithelial membrane antigen) & - & & & \\
\hline CK7 & - & - & - & \\
\hline CK20 & - & - & & \\
\hline $\begin{array}{l}\text { TTF-1 (lung and thyroid; thyroid transcription factor- } 1 \text {, } \\
\text { encoded by Nkx2.1 gene) }\end{array}$ & - & - & & - \\
\hline Napsin (lung and other) & & - & & - \\
\hline $\begin{array}{l}\text { Oct-4 (germ cell; octamer-binding transcription factor } 4 \text {, } \\
\text { also known as POU5F1) }\end{array}$ & & - & & - \\
\hline AFP (yolk sac; alpha-fetoprotein) & & - & & - \\
\hline Glypican-3 (liver, yolk sac) & - & - & - & \\
\hline Hepatocyte (liver) & - & - & & \\
\hline CDX2 (colon; caudal type homeobox 2) & - & & & \\
\hline Villin (colon) & - & & & \\
\hline SDHB (succinate dehydrogenase, subunit B) & & $+($ Normal $)$ & & \\
\hline
\end{tabular}

